\title{
La restauration des bijoux
}

\section{Olivier Tavoso}

\section{(2) OpenEdition}

\section{Journals}

Édition électronique

URL : http://journals.openedition.org/imagesrevues/859

DOI : 10.4000/imagesrevues.859

ISSN : 1778-3801

\section{Éditeur :}

Centre d'Histoire et Théorie des Arts, Groupe d'Anthropologie Historique de l'Occident Médiéval, Laboratoire d'Anthropologie Sociale, UMR 8210 Anthropologie et Histoire des Mondes Antiques

\section{Référence électronique}

Olivier Tavoso, « La restauration des bijoux », Images Re-vues [En ligne], Hors-série 1 | 2008, mis en ligne le 04 mars 2011, consulté le 02 février 2021. URL : http://journals.openedition.org/ imagesrevues/859; DOI : https://doi.org/10.4000/imagesrevues.859

Ce document a été généré automatiquement le 2 février 2021.

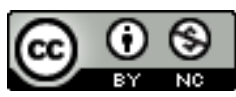

Images Re-vues est mise à disposition selon les termes de la Licence Creative Commons Attribution Pas d'Utilisation Commerciale 4.0 International. 


\title{
La restauration des bijoux
}

\author{
Olivier Tavoso
}

1 La restauration des biens culturels constitués de métal est une discipline dont le champ est très vaste, à la fois matériellement et temporellement. Elle réunit, en effet, des corpus aussi variés que la statuaire monumentale, le patrimoine industriel, l'orfèvrerie ou encore les armes. Elle peut également concerner aussi bien des vestiges datant du $\mathrm{V}^{\mathrm{e}}$ millénaire avant $\mathrm{J}$.-C., époque où est avérée la première métallurgie, que des œuvres contemporaines. La caractéristique commune de ces objets de tous types et de toutes époques est d'être majoritairement ou exclusivement constitués de métaux, matériaux qui présentent des dégradations spécifiques, dont la plus évidente est la corrosion. L'identification et le traitement de ces altérations propres aux métaux relève d'une compétence particulière, qui impose une spécialisation du restaurateur amené à intervenir sur ce corpus.

2 Certains biens culturels majoritairement ou exclusivement constitués de métal peuvent être rassemblés sous le terme générique de « bijoux ». Ce qualificatif désigne des objets différant par le type, la forme ou l'origine géographique, chronologique ou culturelle, mais répondant tous à deux critères. Le premier est l'aspect précieux. Des matériaux et des techniques de fabrication très variés peuvent intervenir dans la réalisation d'un bijou, mais leur mise en œuvre a toujours pour objectif de créer un objet visiblement précieux. Ce dessein préside aux choix des matériaux, dont les qualités esthétiques et la rareté se combinent pour conférer au bijou une valeur immédiatement identifiable par tous. Le second critère est un usage spécifique de ces objets, qui est la parure. Quel que soit le contexte considéré, le port ostentatoire d'un objet précieux confère en effet de la beauté ou du prestige à un individu et, par là, un statut particulier. Le port d'un bijou n'est donc jamais gratuit, il est un signe.

3 L'utilisation d'objets précieux pour l'ornementation personnelle est commune à toutes les sociétés humaines et à toutes les époques. Cette universalité trouve logiquement sa traduction dans les musées, où de nombreux biens culturels, par ailleurs très différents les uns des autres, partagent cette fonction originelle si particulière. Or, il est surprenant de constater que, malgré leurs spécificités, les bijoux conservés dans les collections patrimoniales présentent généralement des altérations similaires et 
caractéristiques. Plus surprenant encore, ces altérations sont manifestement la conséquence d'opérations de "restauration » effectuées sur ces objets. Les pratiques relevées, que l'on peut assimiler à de l'entretien ou même, parfois, à de la réparation pure et simple, signalent l'intervention de personnes n'ayant pas été formées à la conservation et à la restauration. Ces personnes effectuent des opérations dites de "bichonnage ", c'est-à-dire un nettoyage régulier et systématique des objets. Parfois même, des bijoutiers ou des joailliers, artisans maîtrisant le savoir-faire touchant à la fabrication des bijoux, sont encore amenés à intervenir sur des biens culturels intégrés à des collections patrimoniales.

4 Il semble évident à l'examen des objets que ces usages sont la règle, l'exception étant, généralement lorsque le statut de bijou disparaît derrière celui d'objet archéologique ou, parfois, lorsqu'un bijou prestigieux est gravement endommagé, de faire intervenir un spécialiste de la conservation et de la restauration. Cette approche est cependant exceptionnelle et, de fait, les publications spécialisées en conservation et en restauration ne décrivent que très rarement de telles interventions.

5 Les bijoux constituent donc une classe de biens culturels qui échappe encore au champ de la conservation-restauration et ce, paradoxalement, au sein même des musées. Une telle situation n'est certes pas unique. La "conservation-restauration" des biens culturels est une idée moderne et n'est devenue une discipline à part entière que depuis quelques décennies. Dans de nombreux domaines, elle se heurte encore souvent aux habitudes d'entretien et de réparation héritées du passé. On peut, du reste, noter que, parmi les différents organes européens ayant pour mission de former les restaurateurs destinés à intervenir sur les collections patrimoniales, seuls quelques-uns, dont l'Institut national du patrimoine, proposent aujourd'hui un cursus spécialisé en conservation et restauration des biens culturels métalliques.

6 Le sort réservé aux bijoux dans les collections patrimoniales, s'il n'est pas isolé, mérite cependant que l'on s'y attarde. Il permet en effet de mieux comprendre les raisons qui font qu'un corpus constitué d'une immense variété d'objets puisse être envisagé selon une approche unique et traditionnelle et subir, de ce fait, des dégradations importantes. Ces raisons sont à l'évidence liées à la nature même de ces objets, ou plutôt à la perception que le public et les acteurs du monde patrimonial peuvent en avoir. Il semble, en effet, que du classement dans le même concept de bijou, qui est lié à un usage originel commun, découlent des attentes particulières. Les bijoux font ainsi l'objet de certains préjugés. On considère, par exemple, qu'ils sont constitués de matériaux ne s'altérant pas. Une perte d'éclat, de brillance, un changement de couleur dû à la corrosion est donc déroutant et difficile à interpréter. Pourtant, le bijou, lui aussi, s'inscrit dans le temps et subit des altérations : l'or lui-même se corrode, car il est toujours allié à des métaux moins nobles, qui s'oxydent. En outre, beaucoup d'objets sont constitués de métaux sensibles à la corrosion, ayant été simplement dorés ou argentés dans le but d'obtenir l'aspect précieux.

7 Or, une altération, telle qu'une modification liée à la corrosion, engendre une diminution ou une disparition de l'aspect précieux et rend le bijou difficile à identifier immédiatement en tant que tel. L'argent, métal blanc et brillant, devient, lorsqu'il se ternit, noir et sans éclat. Les altérations structurelles importantes, comme la fragmentation ou le bris, nuisent elles aussi à ce que l'on perçoit d'un objet, à ce que l'on peut en comprendre. Dans le cas des bijoux, elles en empêchent virtuellement le port, ce qui entraîne des conséquences pour celui qui observe : chacun connaît l'usage 
d'un collier ou d'un bracelet et il est difficile de ne pas ressentir un trouble devant un objet de ce type présenté fragmenté ou cassé. Les lacunes, quant à elles, sont une perte de matière et une perte d'une partie de la forme qui, ici, est également une perte de matière précieuse. Une bague qui a perdu une pierre a perdu quelque chose d'essentiel, au-delà de sa forme globale et de sa valeur pécuniaire, car elle a également perdu du sens. La trace du « vécu » de l'objet est ici loin d'être perçue comme une valeur ajoutée ou une " patine noble », comme cela peut être le cas d'autres objets métalliques.

Il semble donc que ce qui est essentiel dans la perception immédiate que l'on a des bijoux n'est pas ce qu'ils nous apprennent, mais leur dénominateur commun originel : la préciosité. Cette caractéristique supplante dans les esprits tous les autres aspects du bijou, qu'ils soient historiques, techniques ou archéologiques. Le choix des traitements est donc logiquement guidé par un unique désir, qui est de rétablir, conserver ou même augmenter l'aspect précieux, ce qui engendre, au moment de l'intervention sur les objets, une perte irrémédiable d'information.

Le "bichonnage ", opération courante et en apparence anodine, peut ainsi être à l'origine de dégradations importantes et irréversibles. Tout nettoyage d'une surface métallique est, en effet, synonyme à long terme d'usure de la surface originelle et les produits du commerce couramment utilisés par les personnels des musées s'avèrent souvent très agressifs pour les différents matériaux constitutifs des bijoux.

D'autre part, il faut avoir à l'esprit qu'il est impossible d'appréhender de manière globale un corpus, même homogène en apparence, sans commettre d'erreurs d'interprétation. L'élimination de la corrosion, si gênante pour la compréhension des objets, est de fait souvent la cause de la disparition d'informations capitales. La corrosion de l'or, évènement a priori surprenant concernant ce métal, constitue ainsi une problématique n'ayant fait l'objet que de très rares études. Or, une grande part de notre méconnaissance de ce phénomène, qui intéresse l'archéologie, vient de ce qu'il est exceptionnel de pouvoir étudier un objet archéologique en or n'ayant pas été débarrassé des produits de corrosion qui en masquaient la surface.

11 Plus encore, une approche des bijoux par le biais de l'histoire des techniques et de l'histoire de l'art suffit à démontrer que, depuis l'Antiquité, des traitements de surface intentionnels, visant à modifier l'aspect des métaux précieux, ont été couramment pratiqués. Ces techniques de décor consistaient à donner une teinte au métal, en le corrodant volontairement par des moyens chimiques, ou en imitant l'aspect oxydé à l'aide de glacis colorés.

Des procédés de «mise en couleur » de l'or, que l'on soupçonne sur de nombreux bijoux archéologiques ${ }^{1}$, sont ainsi décrits au Moyen Âge par le moine Théophile², puis à la Renaissance par Benvenuto Cellini ${ }^{3}$. Plus près de nous, les bijoutiers antiquisants de la fin du XIXe siècle en ont fait largement usage. On peut ainsi citer un collier en or et rubis de Louis Wièse conservé au musée des Arts décoratifs, souvent reproduit dans les ouvrages traitant de la bijouterie française de cette époque ${ }^{4}$, dont les parties en creux sont couvertes d'un dépôt rouge-orangé imitant l'aspect caractéristique de l'or issu d'un contexte archéologique.

13 Le ternissement volontaire de l'argent est également une technique de décor attestée dès l'Antiquité. Pline l'Ancien décrit ainsi l'usage égyptien de "colorer » l'argent et s'étonne du prix attaché aux objets ainsi ornés ${ }^{5}$. Il s'agit ici vraisemblablement de niellure, mais l'auteur poursuit en décrivant une autre manière de noircir l'argent, par un procédé bel et bien chimique, ce qui indique que la pratique était, sinon répandue, 
du moins connue dans le monde romain. Ce document est, en revanche, à notre connaissance, la seule description certaine de cette technique. Il semble cependant peu probable que le Moyen Âge et l'époque moderne l'aient ignorée. Le milieu du XIXe siècle marque en revanche un véritable développement du goût pour l'argent terni, où les styles néogothique et néorenaissance l'ont mis à la mode, ce dont il subsiste de nombreux témoignages ${ }^{6}$. François-Désiré Froment-Meurice utilisa, par exemple, fréquemment ce procédé 7 .

Ces différents procédés de décor, qui consistaient à imiter la corrosion naturelle des métaux précieux, ne doivent évidemment pas être confondus avec celle-ci et l'on voit bien grâce à ces exemples qu'aucun traitement de conservation et de restauration ne peut faire l'économie d'un examen diagnostique préalable de chaque objet, qui pourra ainsi révéler ses caractéristiques propres.

La réparation et la transformation de bijoux cassés ou lacunaires, parfois même la " création » de nouveaux objets à partir d'éléments disparates, sont également des pratiques couramment constatées dans les collections patrimoniales. Cet état de fait a été particulièrement vrai entre le milieu du XIX ${ }^{\mathrm{e}}$ siècle et le début $\mathrm{du} \mathrm{XX}^{\mathrm{e}}$, à l'époque des grandes "trouvailles» de trésors archéologiques, mais se rencontre encore aujourd'hui. De fait, toute étude d'un bijou issu d'un contexte archéologique doit aujourd'hui s'accompagner d'une critique d'authenticité, qui est parfois rendue d'autant plus difficile que certains praticiens connaissaient bien les techniques de fabrication antiques et ont pu, à l'exemple du travail des frères Castellani sur les bijoux de la collection Campana, "compléter" ou réparer de façon très crédible des ensembles complets ${ }^{8}$.

Comment, dès lors, le restaurateur peut-il réagir face aux altérations subies par les bijoux? En d'autres termes, comment restaurer les bijoux? Avant tout, il convient de rappeler que le temps passé entre la création d'un bien culturel et le temps présent est par définition incompressible. Ceci implique qu'il est impossible de nier les épisodes vécus par l'objet, ses transformations ou ses dégradations, qui ont modifié définitivement sa matérialité. Ce qui est perdu, de l'aspect et de la matière originale, l'est définitivement. Un nettoyage ou une réparation, même illusionniste, ne servent donc qu'à rendre un objet plus présentable; ils ne peuvent en aucun cas le rendre neuf ou à nouveau complet.

17 La conservation-restauration n'a d'ailleurs pas pour objet de rétablir un bien culturel dans son état d'origine. Son but est d'en assurer la pérennité et de lui restituer une lisibilité suffisante pour qu'il soit à nouveau possible d'en comprendre la forme, le décor et l'usage originel. Des connaissances en chimie, en physique, en histoire de l'art et en histoire des techniques sont pour cela nécessaires. Une approche pluridisciplinaire systématique est, en effet, indispensable à l'appréciation de la complexité de chaque cas et peut seule permettre de réaliser un traitement adapté et répondant à des critères déontologiques d'intervention strictement définis.

Les bijoux ne sauraient être envisagés différemment. Au-delà de ses caractéristiques esthétiques, chaque objet est le véhicule d'informations historiques, techniques et sociologiques. L'étude d'un bijou en or issu d'un contexte archéologique peut ainsi être le moyen, par l'identification des procédés de mise en œuvre du métal, de comprendre le niveau technologique atteint par la société qui l'a produit. Parfois même, l'analyse permet de caractériser les mines ayant fourni le métal ou les gemmes, et de retracer ainsi les échanges commerciaux et culturels entre deux nations disparues. 
19 Les bijoux, comme tous les autres objets patrimoniaux ne doivent donc pas être envisagés, au moment de la restauration, uniquement comme une source de plaisir et de contemplation. La difficulté vient cependant de ce que, dans le cas d'un objet de parure, l'esthétique n'est pas détachable de la fonction. Le bijou est conçu pour être beau et rendre beau et il ne sert qu'à cela. Cependant, s'agissant d'un bien culturel, c'est-à-dire d'un objet dont les particularités font qu'il a été jugé nécessaire de le soustraire du domaine privé pour permettre sa conservation, son étude et son éventuelle intégration dans un ensemble pédagogique, les qualités esthétiques ne constituent qu'une information concernant le statut, la nature, et le sens de l'objet dans son contexte d'origine. Or, cette information, pour essentielle qu'elle soit, ne peut suffire à résumer aucun bijou et l'intervention de conservation-restauration ne doit donc pas consister à rétablir de façon systématique ce statut particulier.

Il est en revanche possible que l'esthétique de l'objet soit identifiée comme une valeur essentielle de celui-ci, qu'il s'agit d'évoquer pour lui permettre de prendre sa place dans un projet muséal. Cette valeur peut alors être privilégiée par rapport à d'autres valeurs qui sont attribuées à l'objet. Ce choix doit cependant être le fruit d'une démarche systématique, préalable à l'intervention, qui aura permis d'en mesurer la complexité et indiquera si l'élimination de la corrosion ou la restitution d'une forme sont possibles sans que l'opération ne soit nuisible à l'intégrité matérielle et à l'intérêt historique de l'objet.

21 Cette démarche est celle qui est couramment suivie aujourd'hui pour la restauration des trésors d'orfèvrerie religieuse, dont l'aspect précieux est là aussi essentiel pour leur compréhension. C'est également la démarche qui préside à la conservationrestauration de tous les biens culturels.

\section{NOTES}

1. Nicolini (G.), Techniques des ors antiques, La bijouterie ibérique du VII au IV siècle, volume 1 , Picard, 1990, p. 47-48.

2. Théophile, Essai sur divers arts, chap. XXXIX.

3. Benvenuto Cellini, Traités de l'orfèvrerie et de la sculpture, chap. XV à XIX.

4. Voir The Belle Epoque of French Jewellry 1850-1910, T. Heneage \& Co, Londres, 1990, cat. 14.

5. Pline, Histoire naturelle, livre XXXIII, paragraphe 131.

6. Rudoe (J.), « Oxidized Silver in the $19^{\text {th }}$ Century: the Documentary Evidence », Metal Plating and Patination: Cultural, Technical and Historical Developments, sous la direction de S. La Niece et P. Craddock, Buttenworth-Heinemann, Londres, 1993, p. 161-170.

7. Voir Trésors d'argent. Les Froment-Meurice, orfêvres romantiques parisiens, Paris, musée de la Vie romantique, 2003, cat. 95, ou The Art of the Jeweller. A Catalogue of the Hull Grundy Gift to the British Museum : Jewellery, Engraved Gems and Goldsmiths' Work, sous la direction de H. Tait, Londres, 1984, cat. 996.

8. Voir à ce sujet le catalogue de l'exposition Castellani and Italian Archeological Jewelry, 2004. 


\section{AUTEUR}

\section{OLIVIER TAVOSO}

Olivier Tavoso est restaurateur du patrimoine (spécialité « arts du feu », métal), diplômé de l'Institut national du Patrimoine. 